\title{
Exodus. Gesetzgebung und Landnahme im kulturellen Gedächtnis Europas
}

\author{
Albrecht Koschorke
}

1.

$\mathrm{Zu}$ den prominentesten Grenzerzählungen der Antike ist ohne Zweifel die Geschichte vom Auszug aus Ägypten zu zählen. Sie beschreibt in mehrfacher Hinsicht ein Schwellengeschehen: räumlich, politisch-konstitutionell, religiös. Das ist so offensichtlich, dass es kaum lohnend scheint, über diesen Tatbestand als solchen viele Worte zu verlieren. Schon die Berufung des Mose, sein Aufenthalt in der Wüste, seine Begegnung mit dem ihm bis dahin unbekannten Gott seiner Väter im brennenden Dornbusch zeigen ihn als Person in einem liminalen Geschehen. Die Verhandlungen mit dem Pharao, die ihn und sein Volk heimsuchenden Plagen, die Vorbereitungen zur Flucht, die Nacht der Erstgeborenentötung - all dies sind Elemente eines Freiheitsmythos, dem geradezu archetypische Qualitäten zukommen. Nicht zufällig erinnert der Durchzug durch das Rote Meer, das dann über den verfolgenden Ägyptern zusammenschlägt, an Motive von Weltranderzählungen. Ein liminales Geschehen anderer Art spielt sich rund um den Berg Sinai ab, an dem das flüchtige Volk der Israeliten Gottes Gesetz und damit zugleich seine kultische Verfassung erhält. Seit der Antike wird Mose unter die großen Gesetzgeber gezählt, und nicht nur in Freuds Deutung erscheint er als der eigentliche Stifter der jüdischen Religion. Auch darin hat die Exodus-Geschichte einen nachgerade archetypischen Rang, zu dem politische und religiöse Neugründungen immer wieder in Parallele gesetzt wurden. Und schließlich hat sich die Verheißung des Landes Kanaan nicht nur als Blaupause für alle möglichen kolonialen Landnahmen interpretieren lassen, sondern ruft auch den Topos vom wiedergefundenen Paradies auf, vergrößert also das Schwellengeschehen in eine eschatologische Dimension.

Die Liste der topischen Elemente, die den biblischen Text zu einem so vielseitig verwendbaren kulturellen Resonanzkörper machen, ist lang: Knechtschaft, die in einen Triumph der Ohnmächtigen umschlägt; Formung eines Volkes unter der Führung einer charismatischen Person, die Mittler zum Göttlichen ist; Überlebensbedingungen im Ausnahmezustand fern der Zivilisation; Ambivalenzen, Zwistigkeiten und Rückschläge innerhalb einer jungen politischen Befreiungsbewegung; Wiederbesinnung auf religiöse Traditionen in Zeiten existentieller 
Krise (beziehungsweise deren Wiedererfindung entsprechend der Formel von der reinvention of tradition); Kodifikationsprozesse, die den Gruppenzusammenhalt auf Dauer stellen sollen; das Drama des Abfalls vom rechten Glauben; Disziplin und Exzess; Aussicht auf ein neues Leben in einem selbstgeschaffenen Gemeinwesen etc. Für all diese - im Einzelnen sehr komplizierten - Prozesse stellt das Buch Exodus eine Erzählvorlage dar, die sich leicht in andere historische Settings verpflanzen lässt: Die Rolle des despotischen Pharao konnte dem Papst oder dem jeweiligen Landesfürsten auf den Leib geschrieben werden; Ägypten mit seinem System der Schuldknechtschaft konnte als Chiffre für die katholische wie für die anglikanische Kirche dienen, aber ebensogut für die Alte Welt als ganze, während in der Neuen Welt ein neues Gelobtes Land verheißen war, ein Neues Israel oder Neues Zion. Die Macht der Analogie war so stark, dass die puritanischen Siedler in den Ureinwohnern Amerikas zuweilen die Kanaaniter wiedererkannten, denen die Israeliten das ihnen von Gott versprochene Land abtrotzen mussten.

Und noch später, in einem eher versteckten Bezug, zeigt sich die Exodus-Erzählung ihre generative, auf andere master narratives übertragbare Kraft. Denn auch die Aufklärung ist, mythologisch gesehen, eine jüngere Schwester des alttestamentlichen Berichts. Dafür bürgt niemand Geringerer als Immanuel Kant, der bekanntlich die Aufklärung als den »Ausgang « - lateinisch: Exodus - »des Menschen aus seiner selbstverschuldeten Unmündigkeit« definierte. Wenn Kant denen, die unaufgeklärt bleiben, »Faulheit und Feigheit « unterstellt und auf ihre Bequemlichkeit schimpft, dann hört er sich wie Moses an, der das Volk Israel aus der ägyptischen Gefangenschaft befreien möchte und mit Zorn erlebt, wie sich die Juden nach den Fleischtöpfen Ägyptens zurücksehnen. Auf subtile Weise setzt sich Kant damit in eine Parallele zu dem Stifter der jüdischen Religion und leitet etwas von der mythischen Wucht der biblischen Erzählung auf das Narrativ des säkularen Projekts der Aufklärung um.

Wenn es auf dieser Tagung also um die Produktivität von Grenzen gehen soll, ist der Grenzraum zwischen Ägypten und Kanaan, in dem sich der Jahr für Jahr an Pessach rituell kommemorierte Gründungsakt des Volkes Israel abspielt, ein paradigmatischer Untersuchungsgegenstand. Statt die aufgelisteten Schwellenmotive und die mit ihnen verbundene Grenzüberschreitungsdynamik abzuarbeiten, möchte ich aber einen anderen Weg gehen und die Aufmerksamkeit auf die Mikrodynamik der im Buch Exodus geschilderten liminalen Vorgänge richten. Denn laut Tagungsexposé soll ja nach der Bedeutung von Grenzen für die Transformation des Wissens gefragt werden. Ich will dies unter Bezug auf erzähl- und gedächtnistheoretische Überlegungen tun, die mit meinen derzeitigen Forschungen über Allgemeine Erzähltheorie zusammenhängen. Und ich will gleich vorausschicken, dass meine Überlegungen kursorisch sind und eher exemplarischen Charakter tragen. ${ }^{1}$

1 In vielen Details stützen sich die folgenden Ausführungen auf den Erkenntnisgewinn zweier 
2.

Meine die folgenden Überlegungen anleitende Grundfrage lautet: Welche Textmerkmale und welche Überlieferungsmechanismen lassen sich als Ursache dafür angeben, dass die Exodus-Geschichte ein über zweieinhalb Jahrtausende hinweg bis heute ungeheuer erfolgreiches master narrative gewesen ist - eine Art narrative Formatierungsvorlage, in die sich kollektive Leidenserfahrungen und Erlösungshoffnungen, politische Befreiungsbewegungen, religiöse Erweckungen und nicht zuletzt alle möglichen kolonialen Unternehmungen einschreiben ließen? Wie funktioniert ein solches Narrativ? Wie kann es so vielen unterschiedlichen Erfahrungen eine Form geben? Was sind die Bedingungen seiner weltweiten Verbreitung, seiner enormen kulturellen Prägekraft?

Die psychologische Erzählforschung hat viele Indizien darüber zusammengetragen, dass Gedächtnisbestände - sowohl auf individueller wie auf kollektiver Ebene - über das Erzählen von merkfähigen Geschichten organisiert werden. Allerdings ist dabei nicht nur der Aspekt des Bewahrens, sondern auch des Ausscheidens und Vergessens bedeutsam. Erzählen ist eine hochgradig selektive Tätigkeit. Es hebt wenige Einzelzüge als signifikant aus einer Masse von Daten heraus. Wie diese Selektion im Einzelnen vor sich geht, hängt unter anderem von den Erfordernissen des jeweiligen Plots ab. Dessen Gestaltung ist aber nicht vollkommen willkürlich; kommunikativ erfolgreiche Plots müssen sich der Voreinstellung der Adressaten anbequemen. Erzähltechnische Experimentreihen ergeben, dass Probanden »easily recognize familiar narratives and unproblematically accept, and even reconstruct, their structure $\ll^{2}$ Läuft eine Geschichte jedoch solchen Vertrautheitserwartungen entgegen, wird sie im Rezeptionsprozess entsprechend umgeschrieben und eingespielten Erzählmustern angepasst. Erzählmuster funktionieren also auf sprachlicher Ebene ähnlich wie kognitive Schemata, die es erlauben, die Überfülle unsortierter empirischer Daten auf typenhafte, leicht wiedererkennbare Formen zurückzuführen, Unbekanntes an Bekanntes zu assimilieren, Abweichungen zu tilgen und überschüssige Details auszublenden. Beides sind Techniken der Komplexitätsreduktion, die zwar zu vielen Fehlleistungen führen, deren großer ökonomischer Vorteil aber darin besteht, dass sie Zeit und Aufwand verringern. Einzelne Erzähltexte können außerordentlich verwickelten Bauplänen folgen; ihre kommunikative Verbreitung und soziale Verhandelbarkeit hängen jedoch davon ab, in welchem $\mathrm{Ma}$ sie dem Grundmuster eines gebräuchlichen Narrativs gehorchen - oder sich nach dessen Vorgaben fehldeuten lassen.

In seiner 1932 erstmals veröffentlichten Pionierstudie Remembering. A Study in Experimental and Social Psychology hat Frederic C. Bartlett die Vergleichbar-

Seminare, die ich in Chicago und Konstanz zusammen mit Jan und Aleida Assmann abgehalten habe. Diesen beiden sei auch an dieser Stelle herzlich gedankt.

2 Czarniawska (2004), 111. 
keit visueller und narrativer Gedächtnistechniken demonstriert. Kettenexperimente mit aus dem Gedächtnis nachgezeichneten Vorlagen zeigen, wie sich der ursprüngliche Gegenstand schrittweise an eine Darstellungskonvention angleicht, die in der Gruppe der Probanden geläufig ist, und zwar vor allem mittels Weglassung und Vereinfachung. Die generelle Tendenz führt also von einem (unidentifizierbaren) visuellen Objekt zum kulturell codierten Piktogramm. Eine besondere Rolle spielt dabei die Neigung, einem amorphen Gebilde durch Namengebung gestalthafte Qualitäten zu leihen und es dann in Angleichung an das benannte Objekt $\mathrm{zu}$ reproduzieren - gegenläufigen empirischen Evidenzen zum Trotz. ${ }^{3}$

Auf analoge Weise kommen Konventionalisierungen im Medium des Erzählens zustande; auch sie sind "produced by a combination of innumerable small changes introduced by a large number of individuals « ${ }^{4}$ Dieser Vorgang ist am besten in Szenarien des Kulturtransfers zu beobachten - dort nämlich, wo »cultural materials coming into a group from outside are gradually worked into a pattern of a relatively stable kind distinctive of that group. The new material is assimilated to the persistent past of the group to which it comes. $\ll^{5}$ Kulturelle Assimilation vollzieht sich zu einem guten Teil durch Anverwandlung fremder Stoffe in die eigenen Erzählmuster, die ihrerseits Ausdruck einer »beharrlichen Vergangenheit« des jeweiligen Kollektivs sind. Das Erzählen wäre hier also weniger ein Transportmittel als ein Filter. Mindestens ebenso wichtig wie seine Mitteilungsfunktion ist seine Eigenschaft, Wissen von geringerer Relevanz oder zu hoher Komplexität nicht weiterzugeben. Es gewinnt seine formende Kraft durch Aussparung dessen, was sich als kommunikativ ungeeignet erweist.

Dem Zweck der Aufwandsminderung dient indessen auch das scheinbar entgegengesetzte Verfahren, unvollständige Schemata den rezeptiven Voreinstellungen entsprechend zu ergänzen. Denn es kostet weniger Aufmerksamkeit und psychische Energie, eine stabile Erwartung bestätigt zu finden, als sich mit Lücken, sperrigen oder regelwidrigen Details aufzuhalten. Auch die Zutat kann eine Ersparnis bedeuten, sofern sie ein bereitstehendes Schema komplettiert. Schemabildung beruht mithin auf drei Grundvorgängen: Verknappung, Angleichung, Vervollständigung. Auf jeden Fall wirkt sie ihrer Haupttendenz nach darauf hin, dass der betreffende Erzählstoff ausgefiltert, geglättet, an genretypische Normalerwartungen angeglichen und dementsprechend verallgemeinerbar wird.

3 Bartlett (1964), 185 und passim.

4 Ebd., 95.

5 Ebd., 280. 
3.

Wendet man die bisher referierten erzählpsychologischen Befunde auf die Exodus-Erzählung an, dann wäre deren Erfolg darin zu suchen, dass sie ein wiederverwendbares Generalschema für gewisse kollektive Grenzerfahrungen und deren erzählerische Bewältigung bereitstellt. Würde man eine Umfrage unter Gläubigen oder mit der Bibel bekannten Nichtgläubigen abhalten, dann würde sich die Schematheorie wahrscheinlich bestätigen: Im Gedächtnis haften die prägnantesten Plot-Sequenzen, von störenden Überfrachtungen oder den Sinn trübenden Querbeziehungen gereinigt, wie ja überhaupt mnemonische und narrative Strukturen gewissermaßen Hand in Hand arbeiten. Das menschliche Gedächtnis ist ein groBer Gleichmacher und Vereinfacher; dementsprechend ist zu erwarten, dass der Anpassungsdruck auf Erzählungen wächst, je häufiger und je länger sie memoriert werden. $\mathrm{Zu}$ dieser Annahme stimmt die Beobachtung, dass mündliche Überlieferungen, die ganz von der Merkfähigkeit ihrer personalen Träger abhängig sind, bekanntlich zu einem stark formularischen, wenn auch individuell variierbaren, Sprachgebrauch neigen.

Allerdings stellen sich einer einfachen Anwendung der Schematheorie auf ein kulturstiftendes Narrativ wie den Exodus zwei Schwierigkeiten entgegen. Erstens entspricht der Text des 2. Buches Mose als solcher in keiner Weise den eben erörterten theoretischen Erwartungen, den man auf einen lange tradierten Gedächtnisstoff richten würde. Und zweitens ist auch seine Rezeption seit der schriftlichen Fixierung des Textes im fünften Jahrhundert v. Chr. keineswegs in den Bahnen einer schematisierenden Aneignung verlaufen.

Was den biblischen Text betrifft, so fallen bei sorgfältigerer Lektüre vor allem sein kompilatorischer Charakter und seine Uneinheitlichkeit auf. Er hat nur oberflächlich etwas mit dem Extrakt gemein, das man in Kinderbibeln von ihm findet. Dieser Sachverhalt lässt sich teilweise erklären, wenn man die Entstehungsbedingungen der Bücher Mose genauer studiert. Wie die Bibelkritik lehrt, geht eine Grundschicht der Mose-Erzählung (Ex. 1-34) auf das 7. Jahrhundert v. Chr. zurück, während die entscheidende Formierungsphase des Textes in die Zeit des Exils (586-539 v. Chr.) fällt. In dieser Zeit und in der Zeit der Rückkehr nach Palästina stand nicht weniger als der Fortbestand des Judentums auf dem Spiel, das durch die Imperien des Zweistromlandes (Assyrer, Babylonier) nicht nur politisch, sondern auch in seiner religiösen und kulturellen Eigenständigkeit bedroht war. Die Alternative war, sich zu assimilieren oder sich unter das Vorzeichen einer exklusiven und widerständigen Gründungserzählung zu stellen. Ein wichtiger Teil dieser Gründungserzählung ist die Mose-Legende, der damit eine außerordentlich politische Bedeutung zukommt. ${ }^{6}$

6 Vgl. Otto (2006), $40 \mathrm{ff} ., 50 f$. 
Die Verfertigung der Bücher Mose findet in einer Umgebung statt, die durch beträchtliche intertextuelle Abhängigkeiten geprägt ist. Nicht zufällig enthält sie zahlreiche Übereinstimmungen mit dem auch aus Griechenland vertrauten antiken Topos des Gesetzgebers, der in einem in Auflösung (anomia) befindlichen Staat eine neue Ordnung, eunomia, etablieren soll - als ein Schwellenheros und Ausnahmewesen, der sich durch sein Genie und Wissen auszeichnet, oft eine Lehrzeit absolviert hat (vorzugsweise im Land der Weisheit, nämlich Ägypten) und überdies für sich in Anspruch nimmt, auf göttlichen Beistand zählen zu können. Mose rückt damit an die Seite von Lykurg, Minos oder Zaleukos, die ihre Gesetze von der Gottheit empfangen oder zumindest so tun - eine Unentschiedenheit, die in Plutarchs Vitae eigens herausgestellt wird. Um der möglichen Konsequenz zu begegnen, dass der von Gott berufene oder sich auf Gott berufende Gesetzgeber als Autor über dem Gesetz stehen würde, wird den Erzählungen vom Gesetzgeber typischerweise eine Episode angefügt, die ihn zur Selbstbestrafung und damit zur Unterwerfung unter sein eigenes Gesetz zwingt, ins Exil schickt oder sterben lässt. ${ }^{7}$ Nur so kann sich das Gesetz von seinem auctor, seinem Urheber, lösen und unabhängige, ewige, unabänderliche Geltung gewinnen. In die Mose-Erzählung ist dieser Aspekt implementiert. Bekanntlich stirbt Mose vor Erreichen des Landes Kanaan, er hat kein Grab und keine Nachfahren, kann also weder Gegenstand eines persönlichen Kults noch Stifter einer Dynastie werden.

Die Bücher Mose haben folglich zum einen Anteil an der politischen Mythologie, die im östlichen Mittelmeerraum des 7. bis 5. Jahrhunderts in Umlauf war. Zum anderen findet ihre Verschriftung und Kanonisierung im Gravitationsfeld von Machtfragen statt, die den Fortbestand Israels und des Judentums in elementarer Weise betreffen. Das verleiht jedem Detail einen sensiblen Charakter. Man wird sich das ähnlich vorstellen dürfen wie bei der Entstehung eines großen Gesetzeswerkes in unseren Tagen: Parteien, Privilegienträger und Interessengruppen streiten um jede Formulierung, um jeden Satz. Hier wird nicht sozusagen gratis fabuliert, sondern um eine Darstellung gerungen, die eine ganze Reihe von Bedürfnissen befriedigen soll: eine Geschichte der Befreiung aus der Knechtschaft erzählen, die erfolgreich zu Ende geführt wird; die Jahwe-Religion als einzige legitime Religion instituieren und zugleich, über einen offenkundigen und keineswegs verhehlten Traditionsriss hinweg, mit dem Glauben der Väter legieren; fremde Kulte vertilgen; die Stammesordnung des Volkes Israel den Verhältnissen der Exilzeit entsprechend retrospektiv zementieren; eine bestimmte politische Struktur durchsetzen beziehungsweise als unvordenklich erscheinen lassen; ein Korpus an Gesetzen und - in der Priesterschrift - an äußerst kleinteiligen Kultvorschriften verbindlich machen; den Anspruch der Juden auf das Land Kanaan sichern und vieles mehr.

7 Szegedy-Maszak (1978). 
Dieser eminent politische Charakter der Torah lässt ein Licht auf die Tatsache fallen, dass die fünf Bücher Mose trotz (oder vielmehr: gerade wegen) ihrer enormen Relevanz in Angelegenheiten der Macht und des Glaubens nicht einfach sozusagen schlank durcherzählt sind, sondern auch so etwas wie eine Geröllhalde ganz unterschiedlicher und teilweise schlecht zusammenpassender Überlieferungen und Erzählstränge darstellen. Wo mehrere Verfasserschaften aufeinandertreffen, divergierende Traditionen bewirtschaftet und unterschiedliche Interessen gewahrt werden wollen, gibt auch der so entstandene Text ein uneinheitliches Bild. Dass sich die kompilatorischen Züge des Bibeltextes an bestimmten Stellen verdichten, ist vor diesem Hintergrund zu erklären. Besonders an die Verkündigung der Zehn Gebote als zentralem Ereignis des Buches Exodus drängeln sich alle möglichen anderen Vorschriftenkataloge heran, die ganz offensichtlich auch Teil an der Aura der Übermittlung des göttlichen Gesetzes haben wollen. Und so werden unmittelbar im Anschluss an den Dekalog - den Gott zuerst mündlich verkündigt, wobei unklar bleibt, ob an Mose oder das ganze Volk (Ex. 19) - detaillierte Altarvorschriften erlassen (aus Erde zu errichten, und wenn steinern, dann unbedingt aus unbehauenen Steinen), werden Regeln für den Handel mit Sklaven, für Eigentums- und Schadensersatzfragen festgelegt, wird die Tötung von Zauberinnen und Zoophilen verlangt und anderes mehr (Ex. 20, 22-26; Ex. 21).

Noch eklatanter ist dieses Verfahren im Zusammenhang mit der Übergabe der Gesetzestafeln an Mose. Wie in einer Suspense-Technik wird zwischen die angekündigte (Ex. 24,12) und die vollzogene (Ex. 31,18) Aushändigung der Tafeln ein langes Textstück mit Anweisungen für die Stiftshütte und ihr Inventar eingeschmuggelt, einschließlich Vorhänge, Teppiche, Räucherwerk und Öl für die Leuchter. Man fühlt sich hier an die pork barrel legislation im amerikanischen Kongress erinnert: An die großen Gesetzesvorhaben werden kleinteilige Partikularvorschriften wie earmarks angeheftet, um bei der Verabschiedung mit durchzugehen.

Wenn man den Aufenthalt am Fuß des Sinai als eine Art Verfassungskonvent der Israeliten betrachtet ${ }^{8}$, dann sind die Inkonsistenzen, die seine Schilderung prägen, ein Ausdruck der widerstrebenden Elemente, die im Akt der Gesetzgebung zusammengebracht werden müssen. Mose soll einerseits die Gesetze von Gott empfangen; er hat privilegierten Zugang zu Gott; er muss aber andererseits als Mensch erscheinen, als Mensch in seiner Ohnmacht, seinem Zorn, sogar seinen privaten Begierden, und nicht als Heiliger. Damit wird der Gefahr vorgebeugt, dass das jüdische Gemeinwesen so etwas wie eine theokratische Schlagseite bekommt. Gott ist einerseits unumschränkter Herr über das Volk Israel, mächtig es zu schützen und zu vernichten; aber er soll andererseits auch mit sich verhandeln lassen, wie er sich schon mit Jakob auf ein Feilschen im orientalischen Stil hatte

8 Vgl. Markl (2007) 
einlassen müssen. Das Volk der Juden ist einerseits Gottes auserwähltes Volk; aber es ist andererseits auch ein murrendes, störrisches, ständig revoltierendes Volk: das verleiht dem biblischen Bericht nicht nur eine sozusagen realistische Note, sondern sichert dem Volk auch eine gewisse Autonomie gegenüber dem Herrschaftsanspruch Moses und der Leviten, letztlich sogar gegen Gott.

Was also wie eine gestalterische Schwäche erscheint - oder als Mangel an glättender, die vorfindlichen Unstimmigkeiten beseitigender Redaktion -, gibt unter diesem Gesichtspunkt dem Pentateuch seine besondere Qualität: Er bringt die inneren Widersprüche und notwendigen Inkohärenzen, das Spiel der Struktur (um von ferne Derrida anzuzitieren ${ }^{9}$ ), das bei der Urszene der gesetzlichen Formung des Staatsvolkes Israel unvermeidlich ist, mit einer unvergleichlichen narrativen Präzision zum Ausdruck. Denn es gibt eine Präzision, die nur Narrative aufbringen, und zwar gerade die verworren scheinenden Narrative. Das Gründungsgeschehen in der Wüste Sinai wird hier als Problem der Vermittlung bearbeitet: als (wackliges, variables) System kommunikativer Verbindungen und Barrieren. Es artikuliert sich in der langwierigen und immer wieder von Rückschlägen geplagten Aushandlung eines Instanzenzuges zwischen Gott, Moses, Aaron, den Ältesten und dem Volk; in den ad-hoc-Maßnahmen der Abtrennung sakraler Zonen auf dem kargen Wüstenboden; und nicht zuletzt in den medialen Verdopplungen und Überlagerungen: Gott donnert für das Volk, während er mit Moses spricht; er spricht die Gesetze, aber er schreibt sie auch. So ist Gott dem Volk abwechselnd nah und fern; er ermächtigt Mose zum Mittler eines politisch-religiösen Bündnisses, ja einer Liebesbeziehung, macht aber gleichzeitig keinen Hehl aus der Distanz, die dabei zu überwinden ist. Dadurch gerät Mose in den Zwiespalt aller Vermittler, nämlich eine Doppelrolle spielen zu müssen - in seinem Fall: einerseits Gottes Sprachrohr und andererseits Unterhändler seines Volkes zu sein. Die Umstände der Niederschrift der Gesetzestafeln bleiben in den verschiedenen Varianten, die das zweite und fünfte Buch Mose bieten, so nebulös, dass sie sich auch für eine Lesart eignen, die Mose als Usurpator göttlicher Gesetzgebungsmacht zeigen. Von dieser Lesart werden vor allem die modernen Mose-Interpreten Gebrauch machen: Zu nennen sind unter anderem Schillers Sendung Moses und Thomas Manns verunglückte Erzählung Das Gesetz. ${ }^{10}$

9 Vgl. Derrida (1985).

10 Friedrich Schiller, Die Sendung Moses; Thomas Mann, Das Gesetz. Thomas Manns Erzählung war als Beitrag zu einer Anthologie gedacht, die nach den Gräueln des Zweiten Weltkriegs und der nationalsozialistischen Gewaltherrschaft den Menschenrechten ein literarisches Denkmal setzen sollte; sie spielt aber mit einer Analogisierung zwischen Moses gewalttätiger Einführung des Gesetzes am Sinai und der Hitler-Diktatur, die dieser textpragmatischen Positionierung zuwider läuft und die Mann-Forschung in Verlegenheit bringt. Vgl. Lubich, (1991). - Zur Mose-Rezeption in der Neuzeit allgemein vgl. Assmann, (1998); Hartwich (1997). 
Konkret wird das Vermittlungs- und Kommunikationsproblem, das mit der Instituierung des Volkes Israel verbunden ist, an den ungeheuren Wegen, die Mose zurücklegen muss. Man beachte, dass er 80 Jahre alt ist, als er von Gott angerufen und mit dem Auszug aus Ägypten betraut wird. Beträchtliche Laufleistung hatte Mose schon in der Eskalationsphase des Konflikts zwischen den jüdischen Zwangsarbeitern und ihren ägyptischen Fronherren zu erbringen; schließlich musste er ja gemeinsam mit Aaron eine Art Pendeldiplomatie zwischen den im Land Gosen, das heißt an der Peripherie Ägyptens siedelnden Hebräern und der Residenz des Pharao betreiben. Noch anspruchsvoller gestaltet sich seine Mission am Berg Sinai, in der Gluthitze der Wüste. Nicht weniger als viermal muss Mose in seinem mühseligen Gesetzgebungsgeschäft auf den Berg Sinai hinaufsteigen, um Gott zu konsultieren, wobei allerdings nur drei Abstiege verzeichnet werden. ${ }^{11}$

4.

Soviel zu der Schwellenerzählung des Buches Exodus in der seinerzeit verschriftlichten Form. Wer ihren Text gründlich und unvoreingenommen studiert, wird schnell erkennen, in welchem Maß sie von Mehrdeutigkeiten, Redundanzen, blinden und stumpfen Motiven durchzogen sind - Hinterlassenschaften einer jahrhundertelangen, vielfach kontroversen Entstehungsgeschichte. Nur in einer höchst selektiven Lektüre, die durch den jeweiligen konfessionellen Kanon gelenkt wird, lassen sich die bekannten Erzählungen aus dem Geröll und Geschiebe der unterschiedlichen Textschichten herauspräparieren und in eine stimmige, von Widersprüchen niemals ganz zu reinigende Form bringen.

Welche Auswirkungen haben die Überdeterminiertheit und Mehrstimmigkeit der Exodus-Erzählung nun, um abschließend zu diesem zweiten Aspekt überzugehen, auf ihre spätere Rezeption? Auch hier kehrt sich, was auf den ersten Blick als Schwäche erscheint, bei näherem Hinsehen in einen Vorteil um. Denn es ist offensichtlich gerade die Polyvalenz des Textes, die den Pentateuch zu einem so ungeheuer fruchtbaren Quelltext gemacht hat, an den sich die unterschiedlichsten Erzähltraditionen anschließen konnten. Michael Walzer hat in seiner klassischen Studie Exodus and Revolution von 1985 dargelegt, wie präzise sich der Unwille des Volkes Israel, sich befreien zu lassen, das Murren und der Hader mit seinen Führern, sein wiederholter Abfall von Gott und Moses Zornhandlungen als das Krisenprotokoll einer revolutionären Situation lesen ließ. ${ }^{12}$ Walzer bezeichnet das Massaker am Berg Sinai, das auf Geheiß Moses nach dem Tanz um das Goldene

11 Nach den (natürlich spielerischen) Berechnungen einer Referatgruppe in dem gemeinsam mit Jan und Aleida Assmann abgehaltenen Mose-Seminar im Wintersemester 2008/2009 legt er dabei ca. 5500 Höhenmeter zurück.

12 Walzer (1988). 
Kalb ausgeführt wurde, als »die erste revolutionäre Säuberung ${ }^{13}{ }^{13}$ Sektenführer und revolutionäre Prediger konnten also gerade auf die Teile des Exodus-Berichts rekurrieren, die sonst aus humanistischem Interesse gern ausgeblendet werden.

Gerade der unsortierte Überschuss des Textes gegenüber seinen konventionalisierenden Lesarten macht ihn also auf vielfältige Weise verwendbar. Christliche Nacherzählungen der Geschichte von den Gesetzestafeln werden die vielen jüdischen Ritualvorschriften, die sich an diesen Schlüsselmoment herandrängen, um an seiner Heiligkeit zu partizipieren, außer Acht lassen; wer die Zehn Gebote in humanistischem Geist als eine Art Magna Charta der Menschheit als ganzer ansieht, wird den Kämpfen des Volkes Israel mit benachbarten Völkerschaften, deren Schilderung viele Kapitel des Buches Exodus füllt, und den dazugehörigen Gemetzeln keine Aufmerksamkeit schenken wollen; aber wo der Auszug aus Ägypten und die Eroberung Kanaans als Blaupause für ein eigenes Landnahmeprojekt verstanden wurde - bei den Puritanern der Neuen Welt oder den Buren Südafrikas -, kam gerade diesen kriegerischen Nebenepisoden eine erhöhte Bedeutung zu. In solchen Situationen wurden sogar die Appelle zum Ethnozid, die der Pentateuch enthält (etwa Deut. 20,17-18), zitier- und predigtfähig.

Daraus lassen sich einige sowohl gedächtnis- als auch erzähltheoretische Schlussfolgerungen ziehen. Es zeigt sich, dass eine der wichtigen funktionalen Eigenschaften des kulturellen Gedächtnisses darin besteht, dass sein Speicher unaufgeräumt ist, dass er weniger einem geordneten Archiv als einer Rumpelkammer gleicht, in der sich alle möglichen oft nutzlos scheinenden Wissens- und Erzählbestände auftürmen. Und es wird ebenso deutlich, dass man es bei den großen Gründungstexten einer Kultur in der Regel nicht mit einfachen, einsträngigen Erzählmustern zu tun hat. Gerade die machtvollen Überlieferungen sind in hohem Maß polymorph. Ihre Stärke besteht offenbar darin, zwischen der Prägnanz einzelner einprägsamer Szenen und Sequenzen auf der einen Seite, andererseits der Unübersichtlichkeit eines tief gestaffelten Fundus von alternativen Erzählanfängen und -resten zu oszillieren.

Diese Polymorphie muss zu dem Mechanismus der schematischen Reduktion, wie er weiter oben beschrieben wurde, nicht im Widerspruch stehen. An jeder Stelle innerhalb der Überlieferungskette mag das Bemühen vorherrschend sein, den Wust der Tradition auf greifbare, dem jeweiligen Verständnishorizont gemäße Vorstellungen und Voreingenommenheiten zurückzuführen. Dieses Bemühen kann jedoch in die entgegengesetzte Richtung ausschlagen, weil zwangsläufig immer ein Teil des Materials gleichsam in unpassende Gussformen gezwungen und dadurch verrätselt, erratisiert wird. Auf der nächsten Rezeptionsstufe wird das unstimmig gewordene und aus seinem bisher unauffälligen Dasein herausgerissene Detail entweder wegretuschiert oder beginnt gerade in seiner Verrätseltheit ein Ei-

13 Ebd., 68. 
genleben. Gerade das Bemühen um semantische Kontrolle kann also dazu führen, dass der Überlieferungsstrom uneinheitlich verläuft, dass an jeder geeigneten Stelle ein Seitenarm abzweigen kann, weil Elemente innerhalb des narrativen Repertoires umgewichtet werden oder weil mitgeführtes, scheinbar überschüssiges Material plötzlich ins Zentrum der Bedeutungsproduktion rückt.

Vor allem die großen, Kultur stiftenden Erzählungen, zumal wenn sie verschriftlicht wurden und dadurch an Umfang gewinnen, schleppen eine Menge Ballast aus früheren Entstehungsphasen mit sich herum; wie bei der DNA lagern sich an die eigentliche und aktuell wirksame Erbinformation Reste der Baupläne früherer Evolutionsstufen oder abweichender Entwicklungspfade an. Man muss also auf zwei Dinge gleichzeitig achten und sie in ihrem Zusammenspiel analysieren: auf die Potenzen des betreffenden Textes; und auf die in der jeweiligen Rezeptionsgegenwart wirksamen Aktivierungsmuster. Wie wir wissen, liefert gerade die Geschichte der Bibel-Exegese viele Beispiele dafür, dass ein für sich genommen unscheinbares Textmerkmal zum Anlass genommen werden konnte, um den betreffenden Text in tiefgreifender Weise umzuwidmen und neuen Deutungsbegehrlichkeiten gefügig zu machen. Und das wiederum gilt für Grenzerzählungen, die Verdichtungspunkte von kulturellen Verhandlungen sind, in einem besonderen Maß.

\section{Literaturverzeichnis}

Assmann, Jan, Moses der Ägypter. Entzifferung einer Gedächtnisspur, München/Wien 1998.

Bartlett, Frederic, Remembering. A Study in Experimental and Social Psychology, zweite Aufl. Cambridge 1964.

Czarniawska, Barbara, Narratives in Social Science Research, London/Thousand Oaks/New Delhi 2004.

Derrida, Jacques, »Die Struktur, das Zeichen und das Spiel im Diskurs der Wissenschaften vom Menschen«, in: Jacqes Derrida, Die Schrift und die Differenz, Frankfurt am Main 1985, $422-442$.

Hartwich, Wolf-Daniel, Die Sendung Moses. Von der Aufklärung bis zu Thomas Mann, München 1997.

Lubich, Frederick, »>Fascinating Faschism $<$. Thomas Manns >Das Gesetz $<$ und seine Selbst-deMontage als Moses-Hitler«, in: German Studies Review 14 (1991), 553-573.

Mann, Thomas, »Das Gesetz«, in: Thomas Mann, Der Tod in Venedig und andere Erzählungen, 60. Aufl. Frankfurt am Main 2004, 259-326.

Markl, Dominik, Der Dekalog als Verfassung des Gottesvolkes, Freiburg i. Br. 2007.

Mose, Eckart Otto, Geschichte und Legende, München 2006.

Schiller, Friedrich, »Die Sendung Moses«, in: Friedrich Schiller, Werke und Briefe in zwölf Bänden, Bd. 6: Historische Schriften und Erzählungen, hg. v. Otto Dann, Frankfurt am Main 2000, 451-474.

Szegedy-Maszak, Andrew, »Legends of the Greek Lawgivers«, in: Greek, Roman and Byzantine Studies 19 (1978), 199-209.

Walzer, Michael, Exodus und Revolution, Berlin 1988. 
